\title{
The expression of glycophorin $A$ and osteoprotegerin is locally increased in carotid atherosclerotic lesions of symptomatic compared to asymptomatic patients
}

\author{
M. HAKIMI ${ }^{1}$, A. HYHLIK-DÜRR ${ }^{1}$, A. VON AU ${ }^{1}$, M. BETZ $^{1}$, S. DEMIREL ${ }^{1}$, \\ S. DIHLMANN ${ }^{1}$, D. BÖCKLER ${ }^{1}$ and M.L. GROSS-WEISSMANN ${ }^{2}$ \\ ${ }^{1}$ Department of Vascular Surgery and ${ }^{2}$ Institute of Pathology, University of Heidelberg, D-69120 Heidelberg, Germany
}

Received February 23, 2013; Accepted April 19, 2013

DOI: 10.3892/ijmm.2013.1401

\begin{abstract}
The aim of this study was to evaluate in detail the histopathological characteristics of endarterectomized carotid atherosclerotic lesions in symptomatic versus asymptomatic patients. Twenty carotid lesions, 10 from asymptomatic and 10 from symptomatic patients who underwent carotid endarterectomy were classified according to histomorphological features. Samples were analyzed for intraplaque localization and for the expression of proteins associated with inflammation, such as CD68, interleukin (IL)-1 $\beta$, tumor necrosis factor- $\alpha$ (TNF- $\alpha)$, pentraxin-3 (PTX-3), nuclear factor- $\kappa \mathrm{B}(\mathrm{NF}-\kappa \mathrm{B})$, $\mathrm{C}$-reactive protein (CRP) and transforming growth factor- $\beta$ (TGF- $\beta$ ), as well as for proteins associated with vascular remodelling, such as matrix-metalloproteinase-9 (MMP-9), glycophorin A (GYPA), osteoprotegerin (OPG), vascular cell adhesion molecule-1 (VCAM-1), endothelin-1 (ET-1), vascular endothelial growth factor (VEGF) and vascular smooth muscle cell actin (VSMA). Corresponding expression scores were compared between the symptomatic and asymptomatic patients and evaluated statistically. The expression of all 14 evaluated markers was significantly elevated in the border zone adjacent to the mixed plaque compared with the unaffected control area of the same sample ( $\mathrm{p}<0.016)$. The expression scores of GYPA and OPG were significantly higher in the border zones around the calcified (GYPA, $\mathrm{p}=0.035$; OPG, $\mathrm{p}=0.043$ ) and mixed (GYPA, $\mathrm{p}<0.001$; OPG, $\mathrm{p}=0.007$ ) plaque zones of symptomatic patients compared to asymptomatic patients. No difference in expression scores was observed for any of the analyzed inflammatory marker proteins between the border zones of symptomatic and asymptomatic patients. In conclusion, the increased expression of GYPA, indicating intraplaque hemor-
\end{abstract}

Correspondence to: Dr Maani Hakimi, Department of Vascular Surgery, University of Heidelberg, Im Neuenheimer Feld 110, D-69120 Heidelberg, Germany

E-mail: maani.hakimi@med.uni-heidelberg.de

Key words: plaque instability, glycophorin A, osteoprotegerin, immunohistochemistry, border zone rhage, and OPG, indicating the transdifferentiation of vascular cells, in carotid atherosclerotic lesions may be associated with an increased risk of plaque instability.

\section{Introduction}

The therapeutic indication of asymptomatic carotid artery stenosis is based on the degree of stenosis, which statistically correlates with the risk of stroke $(1,2)$. According to current concepts, the predominant pathological substrate of cerebral ischemic events results from the embolization of unstable or thrombogenic atherosclerotic plaque. Until now, reliable clinical criteria differentiating between stable and risk prone carotid plaque do not exist.

In addition to extensive image analysis, several biomarkers have been examined, aiming to find predictive factors that indicate an elevated risk of plaque instability and consecutive embolization. Some prominent morphological features, such as vascularization, the amount of necrotic core, thin fibrous cap, intraplaque hemorrhage or increased intima-media thickness (IMT) are associated with plaque vulnerability (3).

However, the cellular events leading to plaque rupture are not yet fully understood. To further characterize the biological activity of plaque and the areas adjacent to plaque, in this study, we assessed the expression and localization of proteins with a potential role in the pathogenesis of atherosclerosis in correlation with qualitatively defined plaque components. In contrast to several other previously described plaque models focusing on plaque area only (4), our study introduces a new model by additionally investigating surrounding border zones neighboring different plaque areas. Plaque from 20 patients that had undergone carotid endarterectomy (CEA) were histologically mapped and stained by immunohistochemistry for marker proteins associated with matrix remodeling, such as matrix-metalloproteinase-9 (MMP-9), glycophorin A (GYPA), osteoprotegerin (OPG), vascular cell adhesion molecule-1 (VCAM-1), endothelin-1 (ET-1) and vascular endothelial growth factor (VEGF), as well as for markers associated with inflammation, such as tumor necrosis factor- $\alpha$ (TNF- $\alpha)$, transforming growth factor- $\beta$ (TGF- $\beta$ ), interleukin-1 $\beta$ (IL-1 $\beta$ ), C-reactive protein (CRP), pentraxin-3 (PTX-3), nuclear factor $-\kappa \mathrm{B}(\mathrm{NF}-\kappa \mathrm{B})$ and $\mathrm{CD} 68$. Vascular smooth muscle cell 
actin (VSMA) was used for the identification of vascular smooth muscle cells (VSMCs).

Emerging evidence indicates that the increased expression of MMP-9 is involved in plaque destabilization (5). MMP-9 belongs to a family of enzymes that promotes the degradation of extracellular matrix components, such as elastin, proteoglycans and laminin and is associated with vascular remodeling and atherogenesis. MMP-9 is produced by macrophages, macrophage foam cells and VSMCs and has been shown to stimulate the production of TNF- $\alpha$ and IL-1, which in turn promotes inflammation (6).

GYPA, a protein specific to erythrocytes, and OPG are considered markers for structural damage. GYPA has been reported to indicate intraplaque hemorrhage and to be associated with necrotic cores and macrophage infiltration in coronary atheromas (7). OPG is a glycoprotein that acts as a receptor for the 'receptor activator of NF- $\mathrm{kB}$ ' ligand. Plasma OPG levels are elevated in patients with vascular disease and the OPG concentration in carotid plaque is associated with the prevalence of artery calcium and plaque instability $(8,9)$.

VCAM-1 plays a role in initiating atherosclerotic cascades by mediating the firm adhesion between macrophages and endothelial cells (10). Its expression in activated endothelial cells is induced by TGF- $\beta$ (11), IL-1 (12) and by cholesterol accumulation (13).

VEGF is a key player in angiogenesis and neovascularization as well as in vascular permeability or thrombogenicity (14). By the initiation of neovascularization, VEGF also plays a role in intraplaque microvessel formation and restenosis (15).

ET-1 is induced in endothelial cells by biomechanical stimuli, hypoxemia, hormones or cytokines. It stimulates the contraction and growth of smooth muscle cells (16).

TNF- $\alpha$ and IL- $1 \beta$ are pro-inflammatory cytokines that are released from activated macrophages. PTX-3 is a soluble receptor of the pattern recognition type, labeling cellular debris and pathogens for phagocytosis by macrophages. Accordingly, it is found in macrophage foam cells which play a key role in atherosclerosis, as well as in circulating blood. Elevated plasma levels of PTX-3 have recently been shown to be associated with plaque vulnerability (17). Elevated plasma levels of CRP induce pro-inflammatory changes by the activation of peripheral leukocytes with the ensuing secretion of plaque-destabilizing mediators, such as MMP-9 (18).

The scavenger receptor, CD68, that interacts with oxidized low-density lipoprotein (oxLDL) amongst others, is a heavily glycosylated transmemberane protein that is predominantly expressed in monocytes and macrophages. Upon interaction with oxLDL, macrophages transform into foam cells. The presence of a high CD68-positive macrophage count has been demonstrated to be associated with plaque destabilization $(19,20)$.

\section{Patients and methods}

Patients. Carotid artery plaque obtained from patients who had undergone CEA was randomly selected from the vascular tissue bank of the Department of Vascular Surgery/National Center for Tumor Diseases (NCT), University of Heidelberg, Heidelberg, Germany. The study population included 20 specimens, 10 from asymptomatic and 10 from
Table I. Patient characteristics and clinical data.

Patients

Items

Asymptomatic Symptomatic

Characteristics

$\begin{array}{lcc}\text { Gender } & & \\ \text { Male } & 7 & 10 \\ \text { Female } & 3 & \\ \text { Mean age (years) } & 68.6 \pm 9.9 & 73.7 \pm 8.7 \\ \text { Mean BMI }\left(\mathrm{kg} / \mathrm{m}^{2}\right) & 26.2 \pm 3.7 & 25.9 \pm 3.8 \\ \text { Mean degree of stenosis }(\%) & 81.5 \pm 8.2 & 81.5 \pm 14.2\end{array}$

Risk factors

Arterial hypertension

$9 \quad 10$

Diabetes mellitus

21

Nicotin

12

Hyperlipidemia

6

8

Medication

Acetylsalicylic acid $\quad 10 \quad 9$

$\begin{array}{lll}\text { Statins } & 6 & 9\end{array}$

Oral antidiabetics/insulin $\quad 2 \quad 1$

Anti-hypertonics

9

BMI, body mass index.

symptomatic patients. Indications for CEA were high-grade internal carotid artery stenosis (as determined by ultrasound) for asymptomatic patients and transient ischemic attack (TIA), amaurosis fugax (AF) or ischemic stroke for symptomatic patients. Neurological events had occurred within 6 months prior to surgery. Clinical data, including medication, blood work and risk factors for atherosclerosis were recorded for all patients (Table I). Patients with atrial fibrillation were excluded. A neurologist examined all symptomatic patients according to clinical standards and the study followed ethical guidelines.

Tissue processing. After dissection of the internal carotid artery from the bifurcation, the intact specimen was harvested by a vascular surgeon (routine eversion technique) (Fig. 1). The fresh carotid eversion specimen was rinsed with saline to remove surface blood, and defined proximal sections were divided into 3 tissue segments (rings of approximately $3 \mathrm{~mm}$ thickness): 2 segments were formalin-fixed and embedded in paraffin according to standard procedures for conventional histological analysis and immunohistochemistry. A third segment was shock-frozen and stored in a freezer at $-80^{\circ} \mathrm{C}$.

Histological graduation. For the assessment of morphological features, the American Heart Association (AHA) classification was adapted for advanced carotid atherosclerotic lesions (21).

Plaque characterization and definition of morphological zones. Based on the morphologial examination, we defined 3 different zones within the atherosclerotic lesions (Fig. 2A): plaque (zone 1), border zone (zone 2) and normal vessel wall 

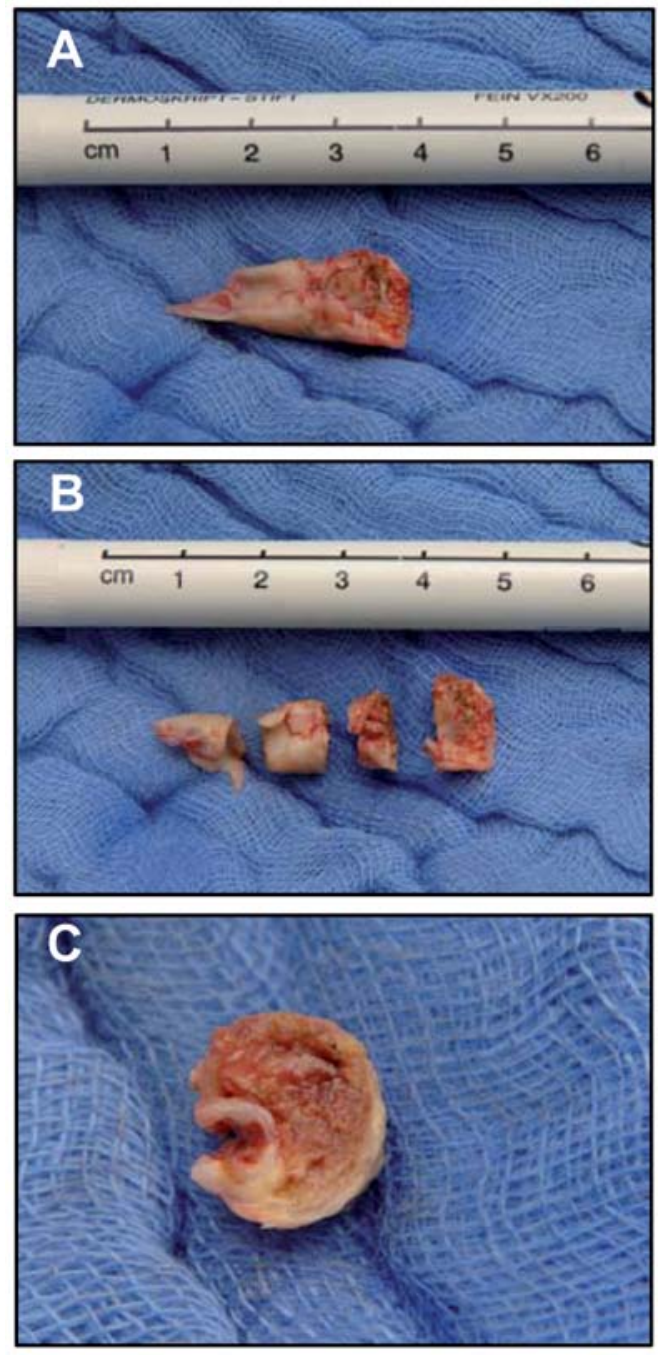

Figure 1. (A) Typical plaque cylinder, harvested using eversion technique. (B) Sliced plaque cylinder. (C) Proximal slice (used for examinations).

(zone 3, control). The plaque zone was divided in 3 major subtypes: calcified (1a), lipid-rich (1b) and mixed type (1c). Similarly, the border zone was classified as bordering to calcified (2a), lipid-rich (2b) or mixed (2c) plaque. The unaffected vessel wall of the same sample (zone 3 ) was used as the control for comparison. To determine the dimension of the different morphological areas, a grid was applied on each transverse sliced sample (square measure of $9033.39 \mu \mathrm{m}^{2}$ per grid unit) (Fig. 2B). Analysis was performed using a microscope (CX40; Olympus, Tokyo, Japan) at an original magnification of x200 and a camera (QImaging, Surrey, BC, Canada). Each grid unit was assigned to a plaque, border or control zone. Corresponding protein expression, as detected by immunohistochemistry, was semi-quantitatively scored by 2 independent investigators unaware of the clinical history of the patient samples (for score evaluations see following section). The plaque zone (zone 1) was characterized by necrotic areas, fibrous tissue, large amounts of calcium and cholesterol. Areas with mixed features demonstrated noticeable calcification and cholesterol-rich areas and signs of surface disruption or fissures were observed (Fig. 2G and H). The main characteristics of zone 2 were large amounts of inflammatory cells, neovascularization and deallocation of the intima and media, while zone 3 represented
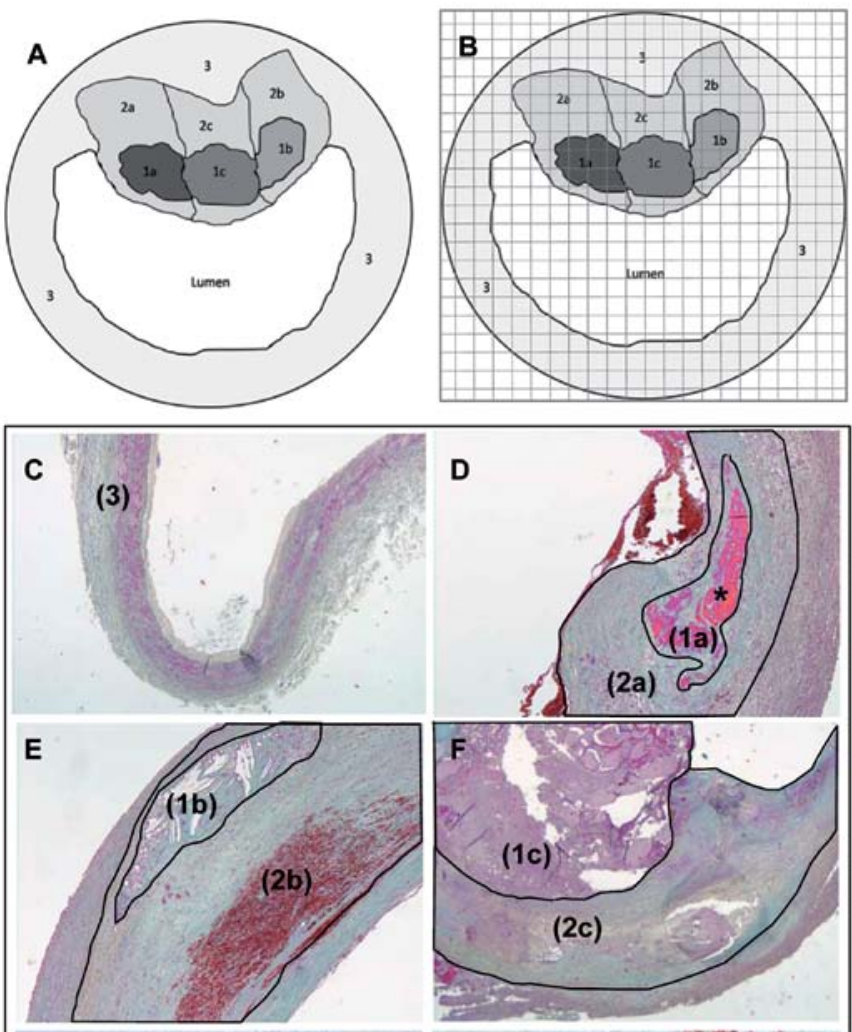

G

(1c)

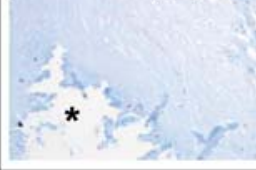

(2c)
H

(1c)
Figure 2. Spatial resolution and zonal mapping of different carotid plaque types. Graphic presentation of (A) zones and (B) grid-units: 1a, calcified plaque; 1b, lipid-rich plaque; 1c, mixed plaque; 2a, border zone to calcified plaque; $2 \mathrm{~b}$, border zone to lipid-rich plaque; $2 \mathrm{c}$, border zone to mixed plaque. (C-H) Representative samples of carotid artery wall exemplifying zonal mapping. (C) Trichrome-stained control zone. (D) Trichrome staining of a calcified plaque and border zone. (E) Trichrome staining showing lipid-rich plaque and border zone. (F) Mixed plaque and border zone. (G) Hematoxylin-stained mixed plaque and border zone; the area of calcification is marked with an asterisk (calcified material is partly detached). (H) Mixed plaque and border zone as shown in $(\mathrm{G})$, immunohistochemically staining for CD68 (red). Original magnification; (C) x20, (D-F) x40, (G and H) x100.

intact vascular tissue with a clear separation of the intima and media plus a significantly lower amount of inflammatory cells (ratio 1:10 compared to zone 2).

\section{Immunohistological analysis}

Staining procedures and antibodies. For the immunohistochemical detection of protein expression, serial $4-\mu$ m-thick sections were prepared from each paraffin-embedded tissue specimen throughout the plaque at the level of the highest degree of stenosis. After deparaffination and rehydration, the samples were pre-treated according to individually optimized protocols (detailed protocols and antibody dilutions are available upon request). The following unconjugated primary antibodies were used for detection: monoclonal mouse anti-MMP-9 was obtained 
from Calbiochem (Merck, Darmstadt, Germany); polyclonal rabbit anti-GYPA, rabbit anti-IL1 $\beta$, rabbit anti-NF- $\kappa \mathrm{B}$, rabbit anti-TGF- $\beta$, monoclonal mouse anti-VCAM, polyclonal rabbit anti-VEGF and polyclonal rabbit anti-OPG were obtained from Santa Cruz (Heidelberg, Germany); rabbit monoclonal antiOPG was purchased from Epigenomics - Biomol (Hamburg, Germany), and monoclonal mouse anti-VSMA was from Sigma (Taufkirchen, Germany); monoclonal mouse anti-CD68 was from USBiological - Biomol (Hamburg, Germany), polyclonal sheep anti-CRP was from Biotrend (Koeln, Germany), polyclonal rabbit anti-ET-1 was from Chemicon (Nuernberg, Germany), polyclonal rabbit anti-TNF- $\alpha$ was from Genzyme (Neu-Isenburg, Germany), and polyclonal rabbit anti-PTX-3 was from Alexis Biochemicals (Loerrach, Germany). The slides were washed $2 \times 5 \mathrm{~min}$ in Tris-buffer and exposed to a biotinylated antibody [MultiLink, HK 340-9K (5\%); BioGenex, San Ramon, CA, USA] for 20 min. After washing again for $2 \times 5$ min in Tris-buffer, the samples were incubated with peroxidase (horseradish peroxidase conjugate) for $20 \mathrm{~min}$, washed again for $2 \times 5$ min in Tris-buffer and stained with Fast Red (Zytomed Systems, Berlin, Germany). Colour development was stopped by the addition of water and the sections were finally counterstained with hematoxylin (Dako REAL, Dako North America, Inc., Capinteria, CA, USA) 1:3 in distilled aqua. For the negative controls, the application of the primary antibody was omitted. Masson-Goldner trichrome staining was used according to standard procedures for the visualization of different connective tissue components.

Expression score. The level of antigen expression was determined semi-quantitatively by a score composed of intensity and quantity. Data for the intensity ran from 0 , no staining; $>1$, faint positive staining and 2 , moderate positive staining to 3 , strong positive staining. Data for the quantity ran from 0 , no staining; $1,<10 \% ; 2,10-50 \% ; 3,51-80 \%$ and $4,>80 \%$ of structures positively stained within the field of view. The score was built by the multiplication of both results $(22,23)$. To avoid observation bias, we first investigated the different zones in all the samples, not discriminating between symptomatic or asymptomatic patients. During a second revision, we focused on the zonal distribution to differentiate between symptomatic and asymptomatic patients.

Statistical analysis. Statistical analysis was performed using SPSS software version 16.0 (SPSS Inc., Chicago, IL, USA). For a comparison of zonal protein scores between border zones and control zones within the same sample, quantitative non-parametric continuous variables were expressed as medians and compared by using the Wilcoxon matched-pairs signed rank test. The Mann-Whitney $U$ test was applied to compare the expression score of each marker derived from different border zones (adjacent to calcified, lipid-rich or mixed plaque) between asymptomatic and symptomatic patients; a value of $\mathrm{p}<0.05$ was considered to indicate a statistically significant difference.

\section{Results}

Patient characteristics. As shown in Table I, the mean age of the patients was $68.6 \pm 9.9$ years for the asymptomatic and $73.7 \pm 8.7$ years for the symptomatic patients. Neurological events of the symptomatic patients had occurred within the last 6 months with a history of recent occurrence of cerebral symptoms [7 patients with non-disabling ischemic stroke (Rankin 1-3), 2 with TIA and 1 with AF]. Nine asymptomatic and 10 symptomatic patients had hypertension, 6 asymptomatic and 8 symptomatic were afflicted with hypercholesterolemia. In total, 3 patients were diabetic and 3 had a history of $>20$ 'pack years' of nicotine abuse. The mean body mass index was $26.2 \pm 3.7 \mathrm{~kg} / \mathrm{m}^{2}$ for the asymptomatic and $25.9 \pm 3.8 \mathrm{~kg} / \mathrm{m}^{2}$ for the symptomatic patients.

Morphological plaque features according to AHA classification. The assessment of morphological plaque features was carried out as previously described in the study by Stary et al (21). As visualized by trichrome staining, all specimens showed severe lesions with calcification, plaque rupture, intra-plaque hemorrhage or fissures equivalent to type $\mathrm{V}$ and VI lesions, according the AHA criteria. Four out of the 10 asymptomatic but only 1 out of the 10 symptomatic patients had type $\mathrm{V}$ lesions. This shows a tendency toward the presence of type VI lesions in symptomatic patients, without statistical significance, however.

Spatial resolution and differentiation of zones with distinctive morphological features. All types of plaque zones and border zones, namely calcified, lipid-rich and mixed type, were observed in the atherosclerotic lesions from the symptomatic and asymptomatic patients. The plaque and border zone accounted for approximately $72 \%( \pm 1.3)$ of the vessel wall area, whereas the control zone accounted for $28 \%( \pm 1.3)$ of the total cross sectional area. Zone 1a (calcified plaque) ranged between 0 and $29 \%$, zone $1 \mathrm{~b}$ (lipid-rich plaque) ranged between 0 and $40 \%$ and zone 1c (mixed plaque) ranged between 25 and $93 \%$ of the plaque area. The same spatial distribution was observed in the border zones. No difference in zonal expansion was observed between the symptomatic or asymptomatic patients.

Zonal distribution of protein expression according to immunohistochemical analysis. In general, all proteins tested were found to be predominantly expressed in the border zones (zone 2), touching or surrounding different plaque types. Except for VSMA, indicating the presence of smooth muscle cells in the vascular media, little protein expression was observed in the control zones (zone 3), whereas almost no protein expression was present within the plaque zones (zone 1). Statistical analysis revealed significantly elevated expression scores of all tested proteins in the border zones compared to control zones. Within the border zones, those touching mixed plaque (zone 2c) presented significantly higher protein expression scores than the border zones touching pure calcified (zone 2a) or pure lipid-rich (zone 2b) plaque (Fig. 3). As indicated by the high VSMA expression, the highest expression of the majority of proteins tested (except for CRP) overlapped with the presence of a high density of smooth muscle cells in zone $2 \mathrm{c}$.

Furthermore, inflammatory markers, such as CD68, NF- $\kappa$ B, IL-1 $\beta$, TNF- $\alpha$ and PTX-3, representing the presence of activated macrophages, displayed significantly higher expression scores in zone $2 \mathrm{c}$ compared to the control zone (Fig. 2G, H and Fig. 3). This indicates that macrophage infiltration is more 

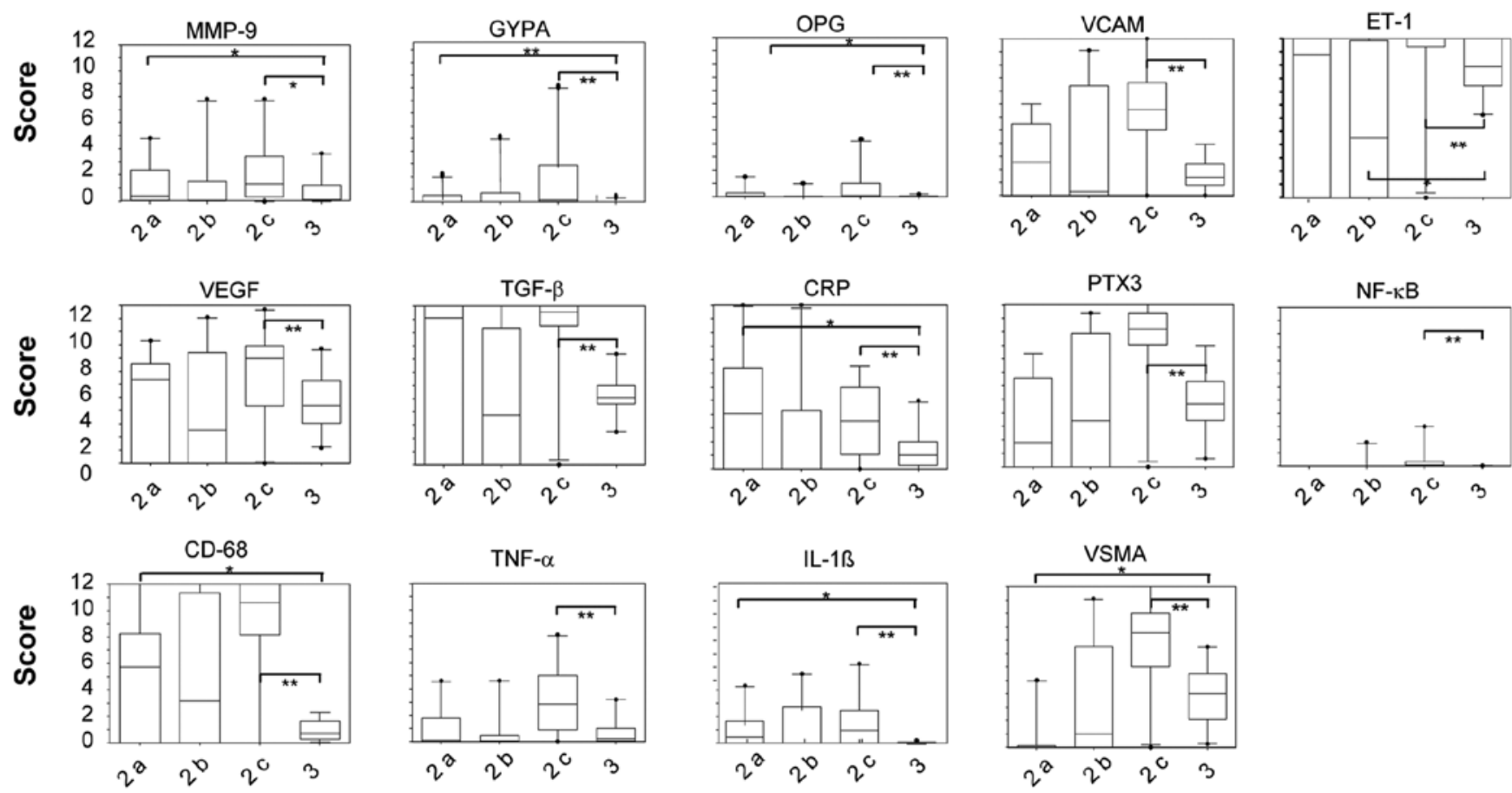

Figure 3. Box-Whisker-Plots showing expression scores derived from immunohistochemical analysis of all carotid lesions, irrespective of patient symptoms. Expression score of zones $2 \mathrm{a}, \mathrm{b}$ and c, respectively, were compared to the control zones. Boxes indicate the median, as well as the upper (75\%) and lower (25\%) quartile. ${ }^{*} \mathrm{p}<0.05 ;{ }^{* *} \mathrm{p}<0.01$.

prominent in the border zones adjacent to mixed plaque than in other border zones.

By contrast, the expression score of the inflammatory protein, CRP, was highest in zone 2 a, touching calcified plaque, followed by border zones touching mixed plaque (zone 2c), its epxression was still significantly higher in these zones compare to the control zones. Of note, CRP was completely absent from the border zone adjacent to lipid-rich plaque (zone 2b). Similar to CRP, the ET-1 expression score was significantly reduced in zone $2 \mathrm{~b}$ compared to the control and other border zones.

Border zones touching calcified plaque (zone 2a) revealed a statistically significant higher expression score of GYPA, MMP-9, OPG, IL-1 $\beta$, CRP and CD68 compared to the control zones (Figs. 3 and 4). In addition to macrophage infiltration, this points to greater intraplaque hemorrhage, matrix degeneration and presumably ongoing calcification in these areas. By contrast, the VSMA expression score was significantly reduced, indicating a reduced number of VSMCs around calcified plaque.

Comparison of protein expression scores between symptomatic and asymptomatic patients. The observation that the expression of inflammatory markers and proteins involved in vascular remodeling was most prominent in the border zones touching mixed plaque, suggested that these plaque types may be more vulnerable. Accordingly, we were interested in whether some of these protein scores differ significantly between symptomatic and asymptomatic patients.

No difference in expression scores was observed for any of the inflammatory marker proteins (CD68, IL-1 $\beta$, NF- $\kappa B$, CRP, PTX-3, TNF- $\alpha$ and TGF- $\beta$ ) between the individual border zones of symptomatic and asymptomatic patients (data not shown).
By contrast, the scores of some marker proteins associated with vessel wall remodeling differed significantly between the asymptomatic and symptomatic patients. The expression scores of GYPA in the border zones around the calcified and mixed plaque areas were slightly but statistically significantly higher in the symptomatic compared to the asymptomatic patients (Fig.5). The same tendency was observed in the border zones around the lipid-rich plaque zones, without reaching statistical significance, however. In addition, the expression scores of OPG were significantly higher in all border zones of symptomatic compared to asymptomatic patients, independent of the adjacent plaque type (Fig. 5). No difference was observed for any of the remaining markers tested (ET-1, MMP-9, VCAM-1, VEGF and VSMA; data not shown).

\section{Discussion}

It is well accepted that atherosclerotic lesions represent complex heterogeneous structures composed of connective tissue, calcification, inflammatory cells and lipids in proportions differing from plaque to plaque (24-26). Increasing evidence suggests that individual plaque morphology and plaque composition contribute to the vulnerability and risk of rupture, in addition to the grade of stenosis $(27,28)$. Consequently, a histopathological staging (AHA classification) was introduced for the classification of atherosclerotic coronary lesions $(21,29)$ that is likewise applied to carotid lesions. Furthermore, a growing number of studies have reported the analysis of single proteins expressed in carotid lesions to be used as biomarkers for the evaluation of plaque rupture and stroke risk at the early stages of disease. However, little progress has been made to achieve this target. In addition, the contribution of these proteins to 

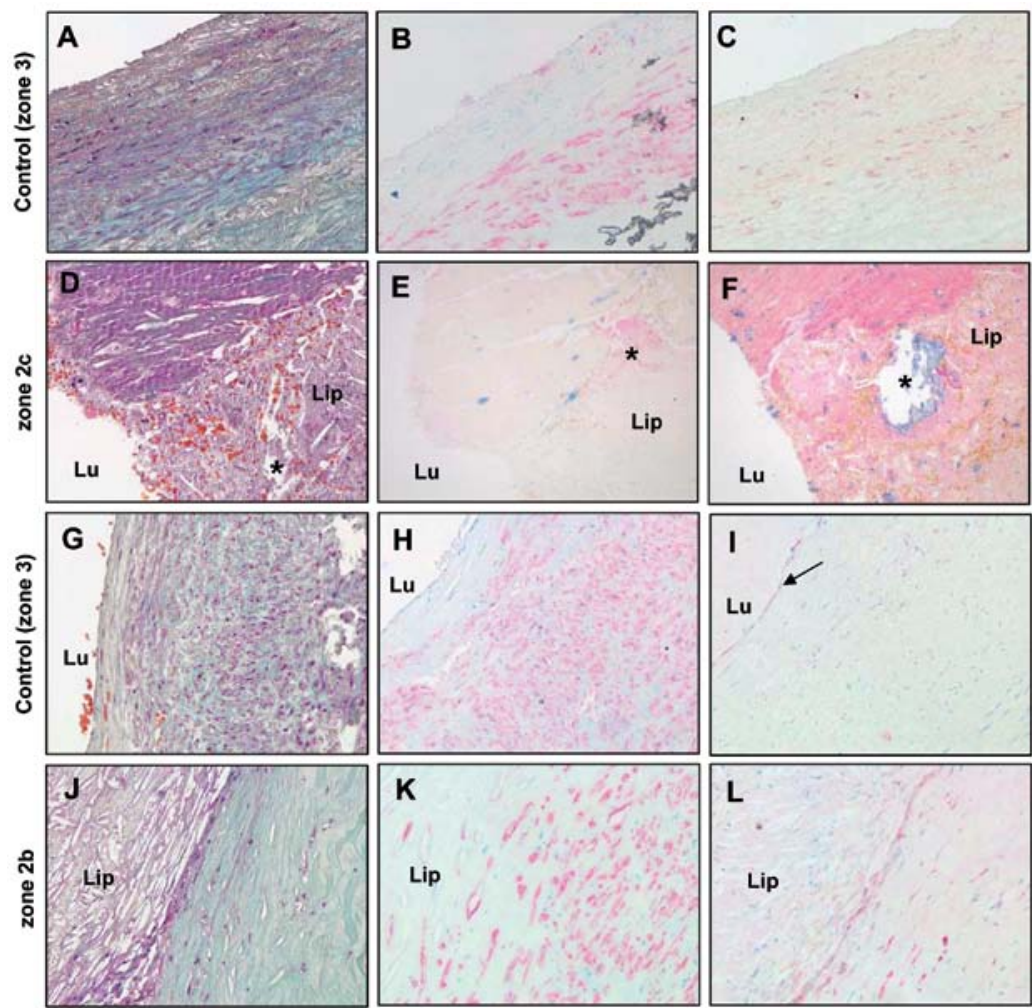

Figure 4. Representative examples of internal carotid artery (ICA) lesions for comparison of plaque border zones with control zones. Original magnification, x200. (A) Trichrome staining of a control zone within an ICA sample derived from a symptomatic patient. (B) Vascular smooth muscle cell actin (VSMA) expression and (C) limited matrix-metalloproteinase-9 (MMP-9) expression in the same area as shown in (A). (D-F) Corresponding mixed plaque zone of the same patient, showing trichrome staining (D), reduced VSMA expression (E) and high MMP-9 expression (F). (G-I) Control zone from the ICA lesion of a second symptomatic patient showing trichrome staining $(\mathrm{G})$, VSMA expression (H) and endothelial vascular cell adhesion molecule (VCAM) expression (I, arrow). (J-L) Corresponding lipid-rich plaque zone of the same sample as in (G-I), showing trichrome staining (J), VSMA expression (K) and increased VCAM expression (L) in the border zone to lipid-rich plaque. "Calcified area; Lu, lumen; Lip, lipid-rich plaque. Expression of individual marker proteins is indicated by red immunohistochemical staining.

A
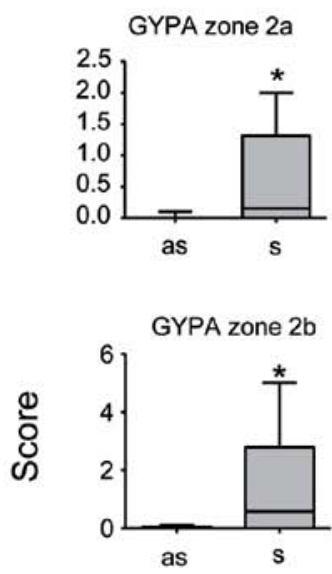

GYPA zone 2c

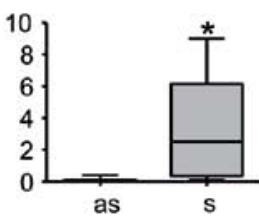

OPG zone 2a

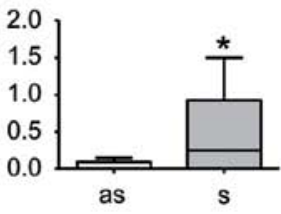

OPG zone $2 b$

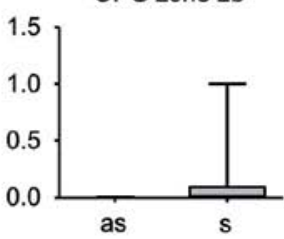

OPG zone 2c

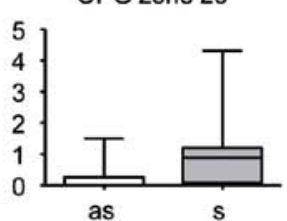

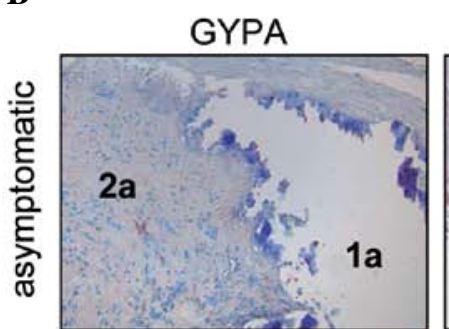
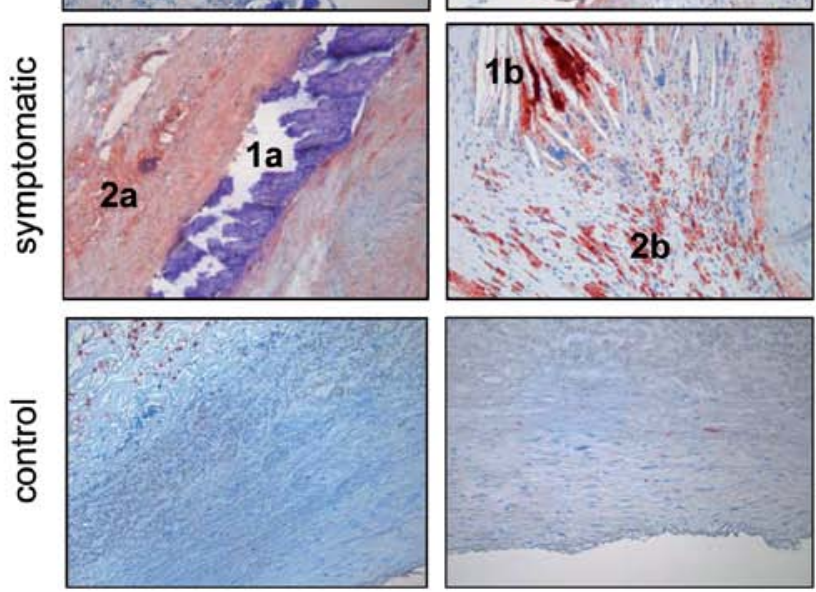

OPG
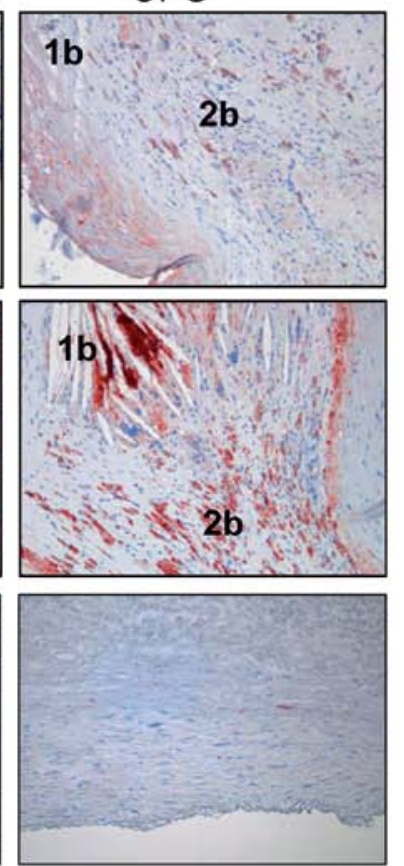

Figure 5. Representative examples of internal carotid artery (ICA) lesions from asymptomatic versus symptomatic patients. (A) Box-Whisker-Plots showing scores of glycophorin A (GYPA) and osteoprotegerin (OPG) expression in plaque border zones of asymptomatic (as) and symptomatic (s) patients, respectively. Boxes indicate the median, as well as the upper (75\%) and lower (25\%) quartile. * $<<0.05$. (B) Immunohistochemical analysis of GYPA and OPG expression (red staining) in carotid lesions derived from asymptomatic and symptomatic patients, respectively. Correspondent immunohistochemical staining of nonatherosclerotic arterial walls is shown for control. Original magnification, x100. 
plaque instability is controversially discussed in the literature and the biological mechanisms leading to plaque rupture are not yet completely understood (30).

To gain deeper insight into carotid plaque composition of asymptomatic and symptomatic patients, in this study, we introduced a new approach to analyze carotid lesions in greater detail by morphologically subdividing the samples into different zones of (cell-free) plaque, border zones touching the plaque material and a control zone. Using this zoning system, we scored the expression of 7 inflammatory marker proteins and 7 proteins associated with remodeling of the artery wall in endarterectomized carotid samples from asymptomatic versus symptomatic patients at punctum maximum of stenosis.

Our findings add to the body of research from previous studies, which predominantly focused on differential gene/ protein expression in carotid lesions as a whole $(27,31)$, or within selected areas, such as calcification (32) or the plaque shoulder without differentiating systematically between plaque components. Moreover, the majority of studies on atherosclerotic plaque heterogeneity and instability have been performed on coronary artery lesions. Although it has been suggested that carotid lesions follow a pattern of development and progression similar to coronary arteries, and may encounter the same mechanisms leading to rupture, this hypothesis is still controversially discussed (25).

Our immunohistochemical analysis revealed a significantly elevated protein expression of all markers investigated in the border zone adjacent to plaque, whereas border zones touching mixed plaque displayed the highest scores. Thus, the area adjacent to a combination of both calcification and lipid deposit showed the highest biological activity with respect to inflammation and tissue remodeling, irrespective of the patient's symptomatic features.

Inflammation is considered a key mechanism in human atherosclerotic plaque vulnerability and disruption (33). However, in our study, the grade of inflammation and macrophage density, as assessed by the expression scores of CD68, IL- $1 \beta$, NF- $\kappa$ B, TNF- $\alpha$, CRP, PTX-3 and TGF- $\beta$, did not differ between the samples from asymptomatic and symptomatic patients. In addition, the expression of markers indicating the recruitment and adhesion of inflammatory cells (VCAM-1), matrix degeneration (MMP-9) and neovascularization (VEGF) was similar in both patient groups.

By contrast, GYPA and OPG expression, despite being a rare event, was significantly more prominent in lesions from our symptomatic patients. Increased levels of GYPA were found in border zones around calcified and mixed plaque in patients who presented with neurological events. The difference was likewise observed in border zones around lipid-rich plaque without reaching statistical significance. Although our results are based on a relatively small sample size, this indicates that intraplaque haemorrhage may be involved in plaque vulnerability. Our data is in line with findings from coronary atheromas, where larger levels of GYPA were associated with advanced plaque instability (7). Moreover, we previously demonstrated that deposits of erythrocyte membrane-derived material (as assessed by GYPA expression) was significantly more pronounced in the coronary intima of patients with chronic renal disease compared to non-renal patients (23). Finally, GYPA expression has recently been shown to correlate with thin fibrous cap and the clinical risk of CEA in a study using a CEA risk classification, which is independent of the symptomatic events if the patients (34). Based on their results, the authors recommended identifying patients with intraplaque haemorrhage by improved imaging and by performing CEA in those patients to avoid neurologic events.

Of note, we identified OPG expression scores to be significantly elevated in all types of border zones in our group of symptomatic patients compared to asymptomatic patients. The role of OPG in vascular calcification and atherosclerosis is not yet clear. Several in vitro studies and animal models have suggested that OPG inhibits vascular calcification, whereas serum OPG levels have been associated with an increased risk of cardiovascular disease in clinical studies (35). OPG has been reported to be expressed in the normal vascular wall by endothelial and VSMCs. Moreover, increased OPG immunoreactivity and mRNA expression have been localized to areas surrounding calcification in the medial layer of Mönckeberg's sclerosis and areas adjacent to calcified neointimal lesions in carotid atherosclerotic arteries (36). Our data, demonstrating an increased OPG expression around different types of plaque not restricted to calcification, point to a more complex mechanism of OPG activity in vascular remodelling that requires further investigation. In particular, it would be of interest to elucidate its role in plaque instability.

In conlcusion, in this study, we present evidence that intraplaque hemorrhage and OPG expression in areas touching mixed plaque that are composed of calcified and lipid-rich components, may be associated with plaque instability and subsequent neurological events in patients with carotid lesions independent of AHA classification. This argues for the need of a more detailed plaque analysis in non-symptomatic patients; for example, by the development of advanced imaging procedures that may aid in the identification of hemorrhage in these patients.

\section{Acknowledgements}

We thank Diana Lutz and Heike Ziebart for their excellent technical assistance in performing immunohistochemical staining. The assistance of Dr Dmitriy Dovzhanskiy in the configuration of the Box-Whisker plots is greatly appreciated.

\section{References}

1. Beneficial effect of carotid endarterectomy in symptomatic patients with high-grade carotid stenosis. North American Symptomatic Carotid Endarterectomy Trial Collaborators. N Engl J Med 325: 445-453, 1991.

2. Halliday AW, Thomas DJ and Mansfield AO: The asymptomatic carotid surgery trial (ACST). Int Angiol 14: 18-20, 1995.

3. Golledge J: Carotid intervention in asymptomatic patients. Stroke 39: e17, 2008.

4. Mauriello A, Sangiorgi GM, Virmani R, et al: A pathobiologic link between risk factors profile and morphological markers of carotid instability. Atherosclerosis 208: 572-580, 2010.

5. Galis ZS, Sukhova GK, Lark MW and Libby P: Increased expression of matrix metalloproteinases and matrix degrading activity in vulnerable regions of human atherosclerotic plaques. J Clin Invest 94: 2493-2503, 1994.

6. Galis ZS and Khatri JJ: Matrix metalloproteinases in vascular remodeling and atherogenesis: the good, the bad, and the ugly. Circ Res 90: 251-262, 2002

7. Kolodgie FD, Gold HK, Burke AP, et al: Intraplaque hemorrhage and progression of coronary atheroma. N Engl J Med 349: 2316-2325, 2003 . 
8. Abedin M, Omland T, Ueland T, et al: Relation of osteoprotegerin to coronary calcium and aortic plaque (from the Dallas Heart Study). Am J Cardiol 99: 513-518, 2007.

9. Golledge J, McCann M, Mangan S, Lam A and Karan M: Osteoprotegerin and osteopontin are expressed at high concentrations within symptomatic carotid atherosclerosis. Stroke 35: 1636-1641, 2004.

10. Adams DH and Shaw S: Leucocyte-endothelial interactions and regulation of leucocyte migration. Lancet 343: 831-836, 1994.

11. Ramana KV, Bhatnagar A and Srivastava SK: Inhibition of aldose reductase attenuates TNF-alpha-induced expression of adhesion molecules in endothelial cells. FASEB J 18: 1209-1218, 2004.

12. Bevilacqua MP: Endothelial-leukocyte adhesion molecules. Annu Rev Immunol 11: 767-804, 1993.

13. Cybulsky MI and Gimbrone MA Jr: Endothelial expression of a mononuclear leukocyte adhesion molecule during atherogenesis. Science 251: 788-791, 1991.

14. Stephan CC and Brock TA: Vascular endothelial growth factor, a multifunctional polypeptide. P R Health Sci J 15: 169-178, 1996.

15. O'Brien ER, Garvin MR, Dev R, et al: Angiogenesis in human coronary atherosclerotic plaques. Am J Pathol 145: 883-894, 1994.

16. Schiffrin EL and Touyz RM: Vascular biology of endothelin. J Cardiovasc Pharmacol 32 (Suppl 3): S2-S13, 1998.

17. Soeki T, Niki T, Kusunose K, et al: Elevated concentrations of pentraxin 3 are associated with coronary plaque vulnerability. J Cardiol 58: 151-157, 2011.

18. Bisoendial RJ, Birjmohun RS, Akdim F, et al: C-reactive protein elicits white blood cell activation in humans. Am J Med 122: 582 e1-e9, 2009.

19. Zeibig S, Li Z, Wagner S, et al: Effect of the oxLDL binding protein Fc-CD68 on plaque extension and vulnerability in atherosclerosis. Circ Res 108: 695-703, 2011

20. Artese L, Ucchino S, Piattelli A, et al: Factors associated with apoptosis in symptomatic and asymptomatic carotid atherosclerotic plaques. Int J Immunopathol Pharmacol 18: 645-653, 2005.

21. Stary HC, Chandler AB, Dinsmore RE, et al: A definition of advanced types of atherosclerotic lesions and a histological classification of atherosclerosis. A report from the Committee on Vascular Lesions of the Council on Arteriosclerosis, American Heart Association. Arterioscler Thromb Vasc Biol 15: 1512-1531, 1995.

22. Remmele W and Stegner HE: Recommendation for uniform definition of an immunoreactive score (IRS) for immunohistochemical estrogen receptor detection (ER-ICA) in breast cancer tissue. Pathologe 8: 138-140, 1987 (In German).
23. Gross ML, Meyer HP, Ziebart H, et al: Calcification of coronary intima and media: immunohistochemistry, backscatter imaging, and x-ray analysis in renal and nonrenal patients. Clin J Am Soc Nephrol 2: 121-134, 2007.

24. Verstraete M: Coronary atherosclerosis and thrombosis. Recenti Prog Med 81: 221-227, 1990.

25. Slevin M, Wang Q, Font MA, et al: Atherothrombosis and plaque heterology: different location or a unique disease? Pathobiology 75: 209-225, 2008.

26. Aidinian G, Weiswasser JM, Arora S, Abularrage CJ, Singh N and Sidawy AN: Carotid plaque morphologic characteristics. Perspect Vasc Surg Endovasc Ther 18: 63-70, 2006.

27. Naghavi M, Libby P, Falk E, et al: From vulnerable plaque to vulnerable patient: a call for new definitions and risk assessment strategies: Part I. Circulation 108: 1664-1672, 2003.

28. Kher N and Marsh JD: Pathobiology of atherosclerosis - a brief review. Semin Thromb Hemost 30: 665-672, 2004.

29. Stary HC, Chandler AB, Dinsmore RE, et al: A definition of advanced types of atherosclerotic lesions and a histological classification of atherosclerosis. A report from the Committee on Vascular Lesions of the Council on Arteriosclerosis, American Heart Association. Circulation 92: 1355-1374, 1995.

30. Koenig W and Khuseyinova N: Biomarkers of atherosclerotic plaque instability and rupture. Arterioscler Thromb Vasc Biol 27: 15-26, 2007.

31. Fagerberg B, Ryndel M, Kjelldahl J, et al: Differences in lesion severity and cellular composition between in vivo assessed upstream and downstream sides of human symptomatic carotid atherosclerotic plaques. J Vasc Res 47: 221-230, 2010.

32. Wahlgren CM, Zheng W, Shaalan W, Tang J and Bassiouny HS Human carotid plaque calcification and vulnerability. Relationship between degree of plaque calcification, fibrous cap inflammatory gene expression and symptomatology. Cerebrovasc Dis 27: 193-200, 2009

33. Libby P, Ridker PM and Hansson GK: Inflammation in atherosclerosis: from pathophysiology to practice. J Am Coll Cardiol 54: 2129-2138, 2009.

34. Hao H, Iihara K, Ishibashi-Ueda $H$, Saito $F$ and Hirota S: Correlation of thin fibrous cap possessing adipophilin-positive macrophages and intraplaque hemorrhage with high clinical risk for carotid endarterectomy. J Neurosurg 114: 1080-1087, 2011.

35. Van Campenhout A and Golledge J: Osteoprotegerin, vascular calcification and atherosclerosis. Atherosclerosis 204: 321-329, 2009.

36. Schoppet M, Al-Fakhri N, Franke FE, et al: Localization of osteoprotegerin, tumor necrosis factor-related apoptosis-inducing ligand, and receptor activator of nuclear factor-kappaB ligand in Mönckeberg's sclerosis and atherosclerosis. J Clin Endocrinol Metab 89: 4104-4112, 2004. 